\title{
Symmetry of Solutions to the Generalized 1-D Optimal Sojourn Time Control Problem
}

\author{
Wei Zhang and Jianghai $\mathrm{Hu}$ \\ School of Electrical and Computer Engineering \\ Purdue University, West Lafayette, IN47906 \\ \{zhang70, jianghai $\} @$ purdue.edu
}

\begin{abstract}
The optimal sojourn time control (OSTC) problem tries to find the feedback control law with reasonable cost that can keep the system state in a certain subset of the state space, called the safe set, for the longest time under random perturbations. The radial symmetry property of its solutions has been proved previously when the state dimension is higher than one. However, the proof does not apply in the one dimensional case. This paper generalizes the OSTC problem by introducing arbitrary weighting functions for both the cost function and the performance measure function, and shows that the solution to the 1-D generalized OSTC problem is radially symmetric. Our proof can also be extended to the multi-dimensional case. Numerical simulation is performed to verify the proof.
\end{abstract}

\section{INTRODUCTION}

Safety is a critical issue in many engineering problems, such as intelligent transportation systems [6], [7], control of Unmanned Aerial Vehicles (UAV) [11], [12], chemical processes, etc. In these applications, the system state is required to stay in a certain subset of the state space called the safe set; and whenever the system state gets into the unsafe set, the system crashes or at least some costly procedure must be invoked to bring the system back to order. Safety of deterministic systems is relatively easy to maintain by properly designed feedback control strategies. However, in practice, systems are often perturbed by random noises, which may steer the state outside the safe set despite the control efforts. Therefore, for such systems, a meaningful problem is to find a feedback control law with a reasonable cost that can keep the randomly perturbed system states in the safe set for as long as possible. This type of control problem is called the optimal sojourn time control (OSTC) problem, and will be the focus of this paper.

While the general stochastic optimal control problem has been widely studied ([8], [13]), the OSTC problem is relatively new. It is first proposed in [1] and is later extended to the general setting of stochastic hybrid systems in [4]. By assuming the symmetry of the solutions, the OSTC problem with one-dimensional state space is simplified and an analytical solution is obtained in [1]. It turns out that the symmetry property becomes even more important in finding the optimal solution for the multi-dimensional case. Hence, before further studying the solutions of the problem, it is necessary to formally prove this property.

By using the symmetrization method [3], the symmetry property is proved when the state dimension $n \geq 2$ in [2].
However, when $n=1$, certain multi-dimensional concepts, such as surface and volume, and some key steps in the proof such as the application of the isoperimetric inequality, are not applicable. Thus an alternative proof for the case $n=1$ is needed. In addition, the OSTC problem [2] assumes that the control cost $J(u)$ is the integral of the $L_{2}$ norm of the control $u$, and the performance measure $W(u)$ to be maximized is just a simple average of the system sojourn time over the safe set. However, for some applications, the control cost may not be equally weighted and the sojourn time in some regions in the safe set may be more important than the others. Thus it is desirable to generalize the OSTC problem to allow some weighting functions for $J(u)$ and $W(u)$.

The contributions of this paper are threefold. Firstly, the generalized OSTC problem is formulated, where the control cost and performance measure are extended to incorporate some weighting functions. Secondly, we prove that with arbitrary symmetric performance weighting and constant control weighting functions, the solution to the one-dimensional generalized OSTC problem is symmetric. Finally, since the way we deal with the newly-introduced performance weighting function does not depend on the state dimension $n$, our proof can be extended to the higher-dimensional case. Thus together with our previous contribution [2], the results of this paper establish completely the radial symmetry property of the solutions to the generalized OSTC problem with an arbitrary state space dimension.

The rest of the paper is organized as follows. In section II, the generalized OSTC problem is formulated. To better present the proof, the problem is transformed into another form in Section III. Section IV reviews some basic definitions and properties of the symmetrization procedures. Then, in Section $\mathrm{V}$, we prove the symmetry property of the 1-dimensional generalized OSTC problem. A numerical example is given in Section VI to verify the results.

\section{Generalized OSTC Problem}

Let $X_{t} \in \mathbb{R}^{n}$ be the state of a dynamical system, and let $\Omega$ be the safe set of the system which is a bounded open connected domain in $\mathbb{R}^{n}$. The derivative of state $X_{t}$ is subject to the perturbations of the white noises $d B_{t} / d t$; and one wants to find a feedback control law $u\left(X_{t}\right)$ that keeps $X_{t}$ in $\Omega$ for the longest time. Mathematically, the above system can 
be described by the following stochastic differential equation

$$
d X_{t}=u\left(X_{t}\right)+d B_{t} .
$$

Define $T=\inf \left\{t \geq 0: X_{t} \notin \Omega\right\}$ as the first exit time of $X_{t}$ from $\Omega$. The expected value of $T$ is denoted by

$$
V(x) \triangleq E^{x}[T]
$$

where $E^{x}$ denotes the expectation taken under the initial condition $X_{0}=x$. Thus $V(x)$ is the expected sojourn time of $X_{t}$ in $\Omega$ starting from $x$. By the standard results of stochastic calculus [9], $V(x)$ is the solution to the PDE

$$
\frac{1}{2} \Delta V(x)+u(x) \cdot \nabla V(x)+1=0, \quad x \in \Omega,
$$

with the boundary condition $\left.V(x)\right|_{\partial \Omega} \equiv 0$. In particular, $V(x)$ is bounded and second order differentiable with piecewise continuous second order derivatives.

As discussed earlier, one wishes to maximize the expected sojourn time $V(x)$ of the state $X_{t}$ in the safe set $\Omega$. Without any constraint on $u$, the problem will obviously result in the trivial solution of an infinite $u$. Thus a well-posed problem is to find a control $u$ with at most certain amount of cost $J(u)$ that can maximize the weighted average of $V(x)$ over the safe set $\Omega$. Compared with [2], the generalized OSTC problem adopts a more general control cost function defined as:

$$
J(u) \triangleq \int_{\Omega} \lambda(x)\|u(x)\|^{2} d x,
$$

and a more general performance function of $u$ defined as:

$$
W(u) \triangleq \int_{\Omega} \mu(x) w(V(x)) d x,
$$

while in [2] only the special case $\lambda(x)=\mu(x)=1$ is studied. Here the weighting functions $\lambda(x)$ and $\mu(x)$ are positive, and $w(\cdot)$ is required to be monotonically increasing on $R_{+}=[0, \infty)$ with $w(0)=0$. For example, $w(x)=$ $x^{k}, x \geq 0$, for some positive integer $k$. Given a control $u$, the sojourn time $V$ is always positive and is the unique solution to the PDE (2). Therefore, $W(u)$ is essentially the weighted average sojourn time of $X_{t}$ over the safe set $\Omega$ under the control $u$, which is a reasonable performance measure.

Given the above definitions, two formulations of the generalized OSTC problem are given below.

Problem 1 (Generalized OSTC Problem): Find the piecewise continuous $u$ that achieves the largest $W(u)$ with an cost $J(u) \leq J_{0}$ for some positive constant $J_{0}$.

Problem 2 (Dual Generalized OSTC Problem): Find the piecewise continuous $u$ with the least cost $J(u)$ subject to $W(u) \geq W_{0}$ for some positive constant $W_{0} \geq W(0)$.

Remark 1: Under zero control $u \equiv 0$, it still takes the state $X_{t}$ some time to exit $\Omega$, i.e., $W(0)>0$. So $W_{0} \geq W(0)$ is required in Problem 2 to avoid trivial solution.

The above two problems are dual to each other, and solving one is equivalent to solving the other [2]. In the rest of this paper, for convenience, we may switch freely between these two formulations.

\section{PROOF SETUP}

We now study Problem 1 with $n=1$, i.e., the onedimensional case. Then $\Omega$ becomes an interval $[-a, a]$ and the sojourn time $V$ is the solution to the ODE

$$
V^{\prime \prime}(x)+2 u(x) V^{\prime}(x)+2=0,
$$

with boundary conditions $V(a)=V(-a)=0$.

Equation (3) gives a one-to-one correspondence between $V$ and $u$. In particular, $u$ can be recovered from $V$ by

$$
u=\frac{\left|1+\frac{1}{2} V^{\prime \prime}\right|^{2}}{\left|V^{\prime}\right|^{2}} \text {. }
$$

Thus to show the symmetry of the solution $u$ to Problem 1, we need only to show the symmetry of the corresponding $V$. To this purpose, we write $J(u)$ in terms of $V$ as:

$$
J(u)=\int_{-a}^{a} \lambda(x) \frac{\left|1+\frac{1}{2} V^{\prime \prime}\right|^{2}}{\left|V^{\prime}\right|^{2}} d x .
$$

This substitution is validated by the following lemma proved in [2].

Lemma 1: Suppose that $V:[-a, a] \rightarrow \mathbb{R}$ is the expected sojourn time corresponding to some optimal control $u$ that solves the 1-D version of Problem 1 (or 2). Then V satisfies

1) $V \geq 0$, and $V(-a)=V(a)=0$;

2) $V$ is second order differentiable with piecewise continuous second order derivatives;

3) $1+\frac{1}{2} V^{\prime \prime} \leq 0$ a.e., and $1+\frac{1}{2} V^{\prime \prime}=0$ whenever $V^{\prime}=0$;

4) $\int_{-a}^{a} \mu(x) \frac{\left|1+\frac{1}{2} V^{\prime \prime}\right|^{2}}{\left|V^{\prime}\right|^{2}} d x<\infty$, for an arbitrary positive integrable function $\mu:[-a, a] \rightarrow \mathbb{R}^{+}$, with $\mu(x) \frac{\left|1+\frac{1}{2} V^{\prime \prime}\right|^{2}}{\left|V^{\prime}\right|^{2}}=0$ at those $x$ where $V^{\prime}=0$.

Part 3) of Lemma 1 guarantees that the substitution (4) is valid for every optimal $V$, namely, the one corresponding to an optimal control $u$. Moreover, if we define $\mathcal{V}([-a, a])$ as the set of all $V:[-a, a] \rightarrow R$ satisfying the above properties, then Lemma 1 implies that we need only to focus on the functions $V$ in $\mathcal{V}([-a, a])$ to find the optimal ones. Hence an equivalent formulation of the Problem 1 in terms of $V$ for the 1-D case is

Problem 3: Find $V \in \mathcal{V}([-a, a])$ that

$$
\begin{aligned}
& \operatorname{maximize} W(u)=\int_{-a}^{a} \mu(x) w(V) d x \\
& \text { subject to } J(u)=\int_{-a}^{a} \lambda(x) \frac{\left|1+\frac{1}{2} V^{\prime \prime}\right|^{2}}{\left|V^{\prime}\right|^{2}} d x \leq J_{0} .
\end{aligned}
$$

Under this setting, the goal of the rest of this paper is to show the following proposition.

Theorem 1: If $\lambda(x)$ is constant, i.e., no control weighting, then for any symmetric function $\mu(x)$, the solution to Problem 3 is also symmetric.

\section{SYMMETRIZATION}

In the calculus of variations, the symmetrization method is a powerful tool to prove that the minimizers of functionals are symmetric functions [3]. Although symmetrization has been widely used in mathematics and physics, it is relatively new to the control society. So before using it in the proof, 
we shall first review some basic concepts and properties of the classical symmetrization method.

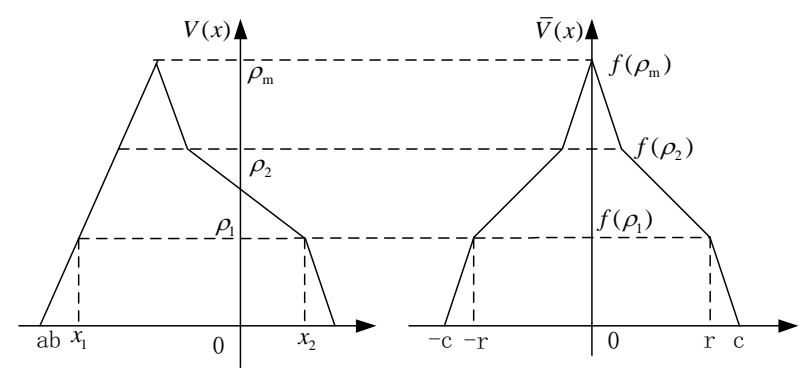

Fig. 1. An example of Schwarz symmetrization.

Roughly speaking, symmetrization is a way of mapping a nonsymmetric function $V(x)$ to a symmetric one $\bar{V}(x)$ while preserving certain properties. Fig. 1 illustrates the geometric idea of symmetrization through a one-dimensional example. Here, $\Omega=[a, b]$ and $V(x) \in\left[0, \rho_{m}\right]$. For each $\rho \in\left[0, \rho_{m}\right]$, denote by $C_{\rho}=\{x \in \Omega \mid V(x)=\rho\}$ the level set of $V(x)$. The symmetrization is performed by symmetrizing the level sets $C_{\rho}$ of $V(x)$. More precisely, for each $\rho \in\left[0, \rho_{m}\right), C_{\rho}$ contains two points $x_{1}$ and $x_{2}$ that may be nonsymmetric with respect to the origin. Define $r=\left|x_{1}-x_{2}\right| / 2$, and let the function $\bar{V}$ take the value $f(\rho)$ at the two symmetric points $\pm r$, where $f:\left[0, \rho_{m}\right] \rightarrow \mathbb{R}^{+}$is some nonnegative function. Typically, $f(\rho)$ is chosen so that the resulting $\bar{V}(x)$ satisfies certain properties, such as preserving certain integrals. If $f(\rho)=\rho$, the above procedure becomes the 1-dimensional Schwarz symmetrization [10], as is the case in Fig. 1. In this case, we denote the resulting symmetric function by $V^{*}(x)$ instead of $\bar{V}(x)$.

More generally, we can define the symmetrization of functions on multi-dimensional spaces as follows.

Definition 1: Let $u: \Omega \rightarrow \mathbb{R}^{+}$be a nonnegative measurable function on $\Omega \subset \mathbb{R}^{n}$. Define $D(\mu)=\{x \in \Omega: u(x) \geq$ $\mu$ \} and denote by $D(\mu)^{*}$ the ball centered at the origin that has the same Lebesgue measure (volume) as $D(\mu)$. If the Lebesgue measure of the set $D(\mu)$ is finite for every $\mu$, then the symmetrization of $u(x)$ is defined as

$$
\begin{aligned}
\bar{u}(x) & =f(\rho(x)) \\
\text { where } \quad \rho(x) & =\sup \left\{\mu: x \in D(\mu)^{*}\right\} .
\end{aligned}
$$

Here $f: \mathbb{R}^{+} \rightarrow \mathbb{R}^{+}$is a nonnegative function.

Remark 2: In the literature, the definition of symmetrization usually does not involve $f$, i.e., $\bar{u}(x)=\rho(x)$. Here we introduce a general function $f$ to be consistent with the proof in Section V. The conventional definition can be thought of as a special case of our definition.

If $f(\rho)=\rho$, the symmetrization is called the Schwarz symmetrization, and has the following properties [10].

Lemma 2: Let $u$ and $g$ satisfy the conditions specified in Definition 1. Denote by the superscript * the Schwarz symmetrization operator of a set or a function. Then,

1) $u^{*}(x)$ is radially symmetric, i.e., $u^{*}(x)=u^{*}(|x|)$;
2) If $\phi(\cdot)$ is a continuous function, then

$$
\int_{\Omega} \phi[u(x)] d x=\int_{\Omega^{*}} \phi\left[u^{*}(x)\right] d x .
$$

3) If $u$ and $g$ are continuous, then

$$
\int_{\Omega} u(x) g(x) d x \leq \int_{\Omega^{*}} u^{*}(x) g^{*}(x) d x
$$

Note that Lemma 2 can not be applied directly in the proof of this paper as the integrand in (5) involves the first and second order derivatives of $V$. Instead, some careful construction of $\bar{V}$ is necessary.

\section{Proof OF SyMmetry}

The basic idea of the proof can be described as follows. For any candidate solution $V \in \mathcal{V}([-a, a])$, we wish to find a symmetric $\bar{V} \in \mathcal{V}([-a, a])$, such that $J(V)=J(\bar{V})$ and $W(V) \leq W(\bar{V})$. If such a $\bar{V}$ always exists for arbitrary $V \in \mathcal{V}([-a, a])$, then the solution to Problem 3 must be symmetric. More precisely, we shall prove the following theorem, which is equivalent to Theorem 1.

Theorem 2: For any function $V \in \mathcal{V}([-a, a])$, there exists a symmetric function $\bar{V} \in \mathcal{V}([-a, a])$, such that

$$
\begin{aligned}
& \qquad \int_{-a}^{a} \frac{\left|1+\frac{1}{2} V^{\prime \prime}\right|^{2}}{\left|V^{\prime}\right|^{2}} d x=\int_{-a}^{a} \frac{\left|1+\frac{1}{2} \bar{V}^{\prime \prime}\right|^{2}}{\left|\bar{V}^{\prime}\right|^{2}} d x, \\
& \text { and } \int_{-a}^{a} \mu(x) w(V) d x \leq \int_{-a}^{a} \mu(x) w(\bar{V}) d x
\end{aligned}
$$

for arbitrary positive symmetric function $\mu(x)$.

The key of the proof of Theorem 2 is the construction of the symmetric function $\bar{V}$ that satisfies the two requirements. According to the discussion in Section IV, this can be done by properly choosing the function $f(\cdot)$. Following this idea, we now go over the symmetrization process to derive the necessary conditions for the desired $f(\cdot)$.

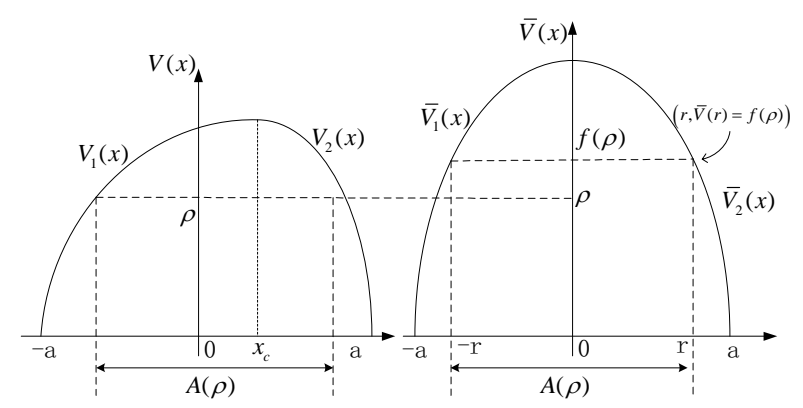

Fig. 2. Symmetrization of V.

According to Lemma $1, V^{\prime \prime} \leq-2<0$. So $V$ is concave on $[-a, a]$. Since $V(a)=V(-a)=0, V$ achieves its maximum value $\rho_{m}$ at a unique point $x_{c}$ in $[-a, a]$, as is shown in Fig. 2. Thus $V^{\prime}\left(x_{c}\right)=0$. Define

$$
\begin{array}{rlrl}
V_{1}(x) & =V(x), & \text { for } x \in\left[-a, x_{c}\right], \\
\text { and } & V_{2}(x) & =V(x), & \text { for } x \in\left[x_{c}, a\right] .
\end{array}
$$


Then $V_{1}$ and $V_{2}$ are monotone functions with inverse

$$
\begin{aligned}
g_{1}(\rho) & =V_{1}^{-1}(\rho) \in\left[-a, x_{c}\right], \\
\text { and } \quad g_{2}(\rho) & =V_{2}^{-1}(\rho) \in\left[x_{c}, a\right], \quad \rho \in\left[0, \rho_{m}\right],
\end{aligned}
$$

respectively. Define $C_{\rho}=\{x \in[-a, a] \mid V(x)=\rho\}$. Then for each $\rho \in\left[0, \rho_{m}\right), C_{\rho}$ consists of two points $x_{1}=g_{1}(\rho)$ and $x_{2}=g_{2}(\rho)$ whose distance is denoted by $A(\rho)$ :

$$
A(\rho)=x_{2}-x_{1}=g_{2}(\rho)-g_{1}(\rho)>0 .
$$

According to the concavity property,

$$
g_{1}^{\prime}(\rho)>0, \text { and } g_{2}^{\prime}(\rho)<0 .
$$

Therefore, $A^{\prime}(\rho)$ can be written as

$$
A^{\prime}(\rho)=-\left(\left|g_{1}^{\prime}(\rho)\right|+\left|g_{2}^{\prime}(\rho)\right|\right)<0 .
$$

Thus to symmetrize $C_{\rho}$, we shall let

$$
r=A(\rho) / 2 \text {, }
$$

and define

$$
\bar{V}(r)=f(\rho),
$$

where $f(\rho)$ is an unknown function to be decided in order for $\bar{V}(r)$ to meet the required conditions in Theorem 2 .

Since the symmetrized function $\bar{V}(r)$ is defined in terms of $\rho$, it is convenient to use change of variables to express the integrals in Theorem 2 in terms of $\rho$. Recall the derivative formula of an inverse function, we have

$$
V_{i}^{\prime}(x)=\frac{1}{g_{i}^{\prime}(\rho)}, \quad i=1,2 .
$$

Furthermore, we can derive that

$$
V_{i}^{\prime \prime}(x)=\frac{d}{d x}\left(\frac{1}{g_{i}^{\prime}(\rho)}\right)=\frac{-g_{i}^{\prime \prime}(\rho) d \rho / d x}{\left[g_{i}^{\prime}(\rho)\right]^{2}}=\frac{-g_{i}^{\prime \prime}(\rho)}{\left[g_{i}^{\prime}(\rho)\right]^{3}} .
$$

Therefore, the integral over $[-a, a]$ on the left-hand side of equation (6) can be splitted on two subintervals to derive

$$
J(V)=\int_{-a}^{a} \frac{\left|1+\frac{1}{2} V^{\prime \prime}\right|^{2}}{\left|V^{\prime}\right|^{2}} d x=\int_{0}^{\rho_{m}} Q(\rho) d \rho
$$

where

$$
Q(\rho)=\frac{\left[2 g_{1}^{\prime}(\rho)^{3}-g_{1}^{\prime \prime}(\rho)\right]^{2}}{4\left|g_{1}^{\prime}(\rho)\right|^{3}}+\frac{\left[2 g_{2}^{\prime}(\rho)^{3}-g_{2}^{\prime \prime}(\rho)\right]^{2}}{4\left|g_{2}^{\prime}(\rho)\right|^{3}} .
$$

In deriving the above two equations, (8) is used to determine the sign of each term.

As for the right-hand side of equation (6), by the symmetry property of $\bar{V}(x)$, we have

$$
J(\bar{V})=2 \int_{0}^{a} \frac{\left|1+\frac{1}{2} \bar{V}^{\prime \prime}\right|^{2}}{\left|\bar{V}^{\prime}\right|^{2}} d r .
$$

From (10) and (11), we can further obtain

$$
\begin{gathered}
d r=\frac{1}{2} A^{\prime}(\rho) d \rho, \\
\bar{V}^{\prime}(r)=\frac{d \bar{V}}{d r}=\frac{d \bar{V}}{d \rho} \frac{d \rho}{d r}=f^{\prime}(\rho) \frac{2}{A^{\prime}(\rho)}, \\
V^{\prime \prime}(r)=\frac{d \bar{V}^{\prime}}{d \rho} \frac{d \rho}{d r}=\frac{d}{d \rho}\left[f^{\prime}(\rho) \frac{1}{A^{\prime}(\rho)}\right] \frac{4}{A^{\prime}(\rho)} .
\end{gathered}
$$

Hence, (12) reduces to

$$
\begin{aligned}
J(\bar{V})=- & \int_{0}^{\rho_{m}} \frac{A^{\prime}(\rho)}{\frac{4}{\left|A^{\prime}(\rho)\right|^{2}}\left[f^{\prime}(\rho)\right]^{2}} \\
& \cdot\left\{1+\frac{2}{A^{\prime}(\rho)} \cdot \frac{d}{d \rho}\left[f^{\prime}(\rho) \frac{1}{A^{\prime}(\rho)}\right]\right\}^{2} d \rho .
\end{aligned}
$$

By equating the integrand in (13) with $Q(\rho)$, we set up the following ODE that the desired $f(\rho)$ needs to satisfy:

$$
\left[1+\frac{2}{\left|A^{\prime}(\rho)\right|} \frac{d}{d \rho}\left(\frac{f^{\prime}(\rho)}{\left|A^{\prime}(\rho)\right|}\right)\right]^{2}=4\left[\frac{f^{\prime}(\rho)}{\left|A^{\prime}(\rho)\right|}\right]^{2} \cdot \frac{Q(\rho)}{\left|A^{\prime}(\rho)\right|} .
$$

Here, we have used (9) to determine the sign of each term.

Besides the ODE (14), in order for the associated $\bar{V}(r)$ to belong to $\mathcal{V}([-a, a]), f(\cdot)$ must satisfy some extra conditions. First of all, since in (11) $\rho=0$ corresponds to $r=a$, and $\bar{V}(a)$ is required to be zero, we must have

$$
f(0)=0 .
$$

In addition, the requirement that $1+\frac{1}{2} V^{\prime \prime} \leq 0$ implies that

$$
\left[1+\frac{2}{\left|A^{\prime}(\rho)\right|} \frac{d}{d \rho}\left(\frac{f^{\prime}(\rho)}{\left|A^{\prime}(\rho)\right|}\right)\right] \leq 0 .
$$

Moreover, we assume that $f(\rho)$ is increasing in $\rho$, i.e.,

$$
f^{\prime}(\rho)>0 \text {. }
$$

Under the above three conditions, the ODE (14) reduces to

$$
y^{\prime}(\rho)+G(\rho) y(\rho)+\frac{1}{2}\left|A^{\prime}(\rho)\right|=0, \quad y(0)=0,
$$

where $y(\rho)=\frac{f^{\prime}(\rho)}{\left|A^{\prime}(\rho)\right|}$, and $G(\rho)=Q(\rho)^{\frac{1}{2}}\left|A^{\prime}(\rho)\right|^{\frac{1}{2}}$. The solution of equation (18) yields $y(\rho)$, which in turn determines $f^{\prime}(\rho)$ as

$$
f^{\prime}(\rho)=\frac{1}{2}\left|A^{\prime}(\rho)\right| e^{-\int_{0}^{\rho} G(\eta) d \eta} \int_{\rho}^{\rho_{m}}\left|A^{\prime}(\xi)\right| e^{\int_{0}^{\xi} G(\eta) d \eta} d \xi
$$

The function $f(\rho)$ can be obtained by integrating $f^{\prime}(\rho)$ :

$$
f(\rho)=\int_{0}^{\rho} f^{\prime}(\chi) d \chi
$$

Since $f(\rho)$ satisfies the conditions (15), (16), and (17), the $\bar{V}$ constructed in (11) belongs to $\mathcal{V}([-a, a])$ and satisfies the condition (6). Thus the only thing left now is to show that $\bar{V}$ also satisfies condition (7). To this purpose, we introduce the following lemma.

Lemma 3: The $P(\rho)$ defined as follows

$$
P(\rho)=\frac{1}{\left|g_{1}^{\prime}(\rho)\right|}+\frac{1}{\left|g_{2}^{\prime}(\rho)\right|}
$$

has the following properties:

1) $P(\rho)\left|A^{\prime}(\rho)\right| \geq 4$;

2) $P\left(\rho_{m}\right)=0$

3) $Q(\rho)^{\frac{1}{2}} P(\rho)^{\frac{1}{2}} \geq A^{\prime}(\rho)-\frac{1}{2} P^{\prime}(\rho)$.

Proof: The first two are obvious, thus we only prove the third one. By Cauchy-Schwarz inequality, the following is true for nonnegative numbers $p_{i}$ 's and $q_{i}$ 's.

$$
\left(p_{1}^{2}+p_{2}^{2}\right)\left(q_{1}^{2}+q_{2}^{2}\right) \geq\left(p_{1} q_{1}+p_{2} q_{2}\right)^{2} .
$$


Hence, we have

$$
[Q(\rho) P(\rho)]^{\frac{1}{2}} \geq\left|\frac{2 g_{1}^{\prime}(\rho)^{3}-g_{1}^{\prime \prime}(\rho)}{2 g_{1}^{\prime}(\rho)^{2}}\right|+\left|\frac{2 g_{2}^{\prime}(\rho)^{3}-g_{2}^{\prime \prime}(\rho)}{2 g_{2}^{\prime}(\rho)^{2}}\right| .
$$

Integrating both sides over $\left[\rho_{1}, \rho_{2}\right] \subset\left[0, \rho_{m}\right]$, we have

$$
\begin{gathered}
\int_{\rho_{1}}^{\rho_{2}}[Q(\rho) P(\rho)]^{\frac{1}{2}} d \rho \geq-\int_{\rho_{1}}^{\rho_{2}} \frac{2 g_{1}^{\prime}(\rho)^{3}-g_{1}^{\prime \prime}(\rho)}{2 g_{1}^{\prime}(\rho)^{2}} d \rho \\
+\int_{\rho_{1}}^{\rho_{2}} \frac{2 g_{2}^{\prime}(\rho)^{3}-g_{2}^{\prime \prime}(\rho)}{2 g_{2}^{\prime}(\rho)^{2}} d \rho \\
=-\int_{\rho_{1}}^{\rho_{2}}\left[g_{1}^{\prime}(\rho)-g_{2}^{\prime}(\rho)\right] d \rho-\frac{1}{2} \int_{\rho_{1}}^{\rho_{2}}\left[\frac{g_{1}^{\prime \prime}(\rho)}{g_{1}^{\prime}(\rho)^{2}}-\frac{g_{2}^{\prime \prime}(\rho)}{g_{2}^{\prime}(\rho)^{2}}\right] d \rho \\
=\left[A\left(\rho_{2}\right)-A\left(\rho_{1}\right)\right]-\frac{1}{2}\left[P\left(\rho_{2}\right)-P\left(\rho_{1}\right)\right],
\end{gathered}
$$

where in deriving the first inequality we use (8) and the fact that

$$
\frac{2 g_{i}^{\prime}(\rho)^{3}-g_{i}^{\prime \prime}(\rho)}{2 g_{i}^{\prime}(\rho)^{3}}=1+\frac{1}{2} V_{i}^{\prime \prime}(x) \leq 0, \quad i=1,2 .
$$

Therefore, the desired result can be obtained by dividing both sides of (22) by $\rho_{2}-\rho_{1}$ and letting $\rho_{2} \rightarrow \rho_{1}$.

Lemma 3 can be used to prove an important property of the function $f(\rho)$ defined in (20).

Lemma 4: The solution $f(\rho)$ to the ODE (14) satisfies $f^{\prime}(\rho) \geq 1$ for all $\rho \in\left[0, \rho_{m}\right)$.

Proof: For any $\rho \in\left[0, \rho_{m}\right)$, by part 1) of Lemma 3, we have

$$
\frac{1}{4} P(\rho) G^{2}(\rho)=\frac{1}{4} P(\rho)\left|A^{\prime}(\rho)\right| Q(\rho) \geq Q(\rho) .
$$

Taking square root, multiplying both sides by $P^{\frac{1}{2}}(\rho)$ and applying part 3) of Lemma 3, we have

$$
\frac{1}{2} P(\rho) G(\rho) \geq P^{1 / 2}(\rho) Q^{1 / 2}(\rho) \geq A^{\prime}(\rho)-\frac{1}{2} P^{\prime}(\rho),
$$

which implies

$$
\begin{gathered}
\frac{1}{2}\left|A^{\prime}(\rho)\right| e^{\int_{0}^{\rho} G(\eta) d \eta}=-\frac{1}{2} A^{\prime}(\rho) e^{\int_{0}^{\rho} G(\eta) d \eta} \\
\geq \frac{1}{4}\left[-P^{\prime}(\rho)-P(\rho) G(\rho)\right] e^{\int_{0}^{\rho} G(\eta) d \eta} .
\end{gathered}
$$

Hence,

$$
\begin{aligned}
& \int_{\rho}^{\rho_{m}} \frac{1}{2}\left|A^{\prime}(\xi)\right| e^{\int_{0}^{\xi} G(\eta) d \eta} d \xi \\
& \geq \int_{\rho}^{\rho_{m}} \frac{1}{4}\left[-P^{\prime}(\xi)-P(\xi) G(\xi)\right] e^{\int_{0}^{\xi} G(\eta) d \eta} \\
& =\left.\frac{1}{4}\left[-P(\xi) e^{\int_{0}^{\xi} G(\eta) d \eta}\right]\right|_{\xi=\rho} ^{\xi=\rho_{m}}=\frac{1}{4} P(\rho) e^{\int_{0}^{\rho} G(\eta) d \eta},
\end{aligned}
$$

where we have used part 2) of Lemma 3. Then by the expression of $f^{\prime}(\rho)$ in (19), we have

$$
\begin{aligned}
f^{\prime}(\rho) & =\frac{1}{2}\left|A^{\prime}(\rho)\right| e^{-\int_{0}^{\rho} G(\eta) d \eta} \int_{\rho}^{\rho_{m}}\left|A^{\prime}(\xi)\right| e^{\int_{0}^{\xi} G(\eta) d \eta} d \xi \\
& \geq\left|A^{\prime}(\rho)\right| e^{-\int_{0}^{\rho} G(\eta) d \eta} \cdot \frac{1}{4} P(\rho) e^{\int_{0}^{\rho} G(\eta) d \eta} \geq 1,
\end{aligned}
$$

which is the desired result.

With Lemma 4, we are ready to prove Theorem 2.
Proof: [Proof of Theorem 2] Given an arbitrary $V \in$ $\mathcal{V}([-a, a])$, let $\bar{V}(r)=f(\rho)$, where $r$ and $\rho$ are related by (10), and $f(\rho)$ is defined by (20). The way we compute $f$ guarantees that $\bar{V} \in \mathcal{V}([-a, a])$ and $\bar{V}$ satisfies (6). Moreover, $f(0)=0$, and by Lemma $4, f^{\prime}(\rho) \geq 1$, which implies that $f(\rho) \geq \rho$. Therefore,

$$
\begin{aligned}
& W(\bar{V})=\int_{-a}^{a} \mu(x) w(\bar{V}) d x \\
= & \int_{0}^{\rho_{m}} \mu(A(\rho) / 2) w[f(\rho)]\left(\left|g_{1}^{\prime}(\rho)\right|+\left|g_{2}^{\prime}(\rho)\right|\right) d \rho \\
= & \int_{0}^{\rho_{m}} \mu(A(\rho) / 2) w[f(\rho)]\left|A^{\prime}(\rho)\right| d \rho \\
\geq & \int_{0}^{\rho_{m}} \mu(A(\rho) / 2) w(\rho)\left|A^{\prime}(\rho)\right| d \rho \\
= & \int_{-a}^{a} \mu^{*}(x) w\left(V^{*}\right) d x=\int_{-a}^{a} \mu^{*}(x) w^{*}(V) d x,
\end{aligned}
$$

where we have used the symmetric property of $\mu(x)$, and the nondecreasing property of $w(x)$. The last step of the above derivation follows directly from the definition of the Schwarz symmetrization, which preserves the function value during the symmetrization process. Applying part 3) of Lemma 2 to (23), the desired result can be obtained:

$$
\begin{aligned}
W(\bar{V}) & \geq \int_{-a}^{a} \mu^{*}(x) w^{*}(V) d x \\
& \geq \int_{-a}^{a} \mu(x) w(V) d x=W(V) .
\end{aligned}
$$

Thus $\bar{V} \in \mathcal{V}([-a, a])$ and $\bar{V}$ satisfies both (6) and (7). This completes the proof of the theorem.

As a result, Theorem 1 is also proved.

In summary, the proof of Theorem 2 consists of two parts. The first part proves that the $f(\rho)$, whose corresponding $\bar{V}$ satisfies (6), has the property $f(\rho) \geq \rho$. The second part uses this result and the property of the Schwarz symmetrization (Lemma 2) to derive the inequality (7). The properties in Lemma 2 hold for an arbitrary Euclidean space, thus only the first part of the proof depends on the state dimension $n$. Since [2] proves that the $f(\rho)$, corresponding to the symmetrization of multi-dimensional state space $(n \geq 2)$, satisfies $f(\rho) \geq \rho$, the following theorem follows immediately.

Theorem 3: Suppose that the safe set $\Omega$ is radially symmetric. If $\lambda(x)$ is constant over $\Omega$ and $\mu(x)$ is a positive radially symmetric function defined on $\Omega$, the solution to the general OSTC problem (Problem 1 or 2) is radially symmetric for an arbitrary state dimension $n$.

\section{NUMERICAL VERIFICATION}

In this section, a numerical example is given to verify our proof in Section V. To this end, we will numerically solve a 1-D OSTC problem without assuming the symmetry property to see whether the solution is symmetric. In particular, we focus on Problem 2.

The goal is to find a control $u$ that minimizes $J(u)$ subject to the 1-D PDE (3) and $\int_{-a}^{a} \mu(x) w(V) d x=W_{0}$. To deal 
TABLE I

SiMUlation PARAMETERS

\begin{tabular}{|c|c|c|c|c|c|c|}
\hline Para. & $a$ & $\lambda(x)$ & $\mu_{1}(x)$ & $\mu_{2}(x)$ & $w(V)$ & $W_{0}$ \\
\hline Value & 1 & 1 & $10|x|+1$ & $5|x|+1$ & $V$ & 10 \\
\hline
\end{tabular}

with the constraints, define $y_{1}=\int_{-a}^{x} \mu(x) w(V) d x, y_{2}=V$, and $y_{3}=y_{2}^{\prime}$. Then the 1-D generalized OSTC problem is equivalent to the following optimal control problem:

Problem 4: Find $u$ that

$$
\begin{aligned}
\text { Minimize } & \int_{-a}^{a} \lambda(x) u^{2}(x) d x \\
\text { Subject to } & \left\{\begin{array}{l}
y_{1}^{\prime}(x)=\mu(x) w\left(y_{2}(x)\right) \\
y_{2}^{\prime}(x)=y_{3}(x) \\
y_{3}^{\prime}(x)=-2 u(x) y_{3}(x)-2
\end{array}\right.
\end{aligned}
$$$$
y_{1}(-a)=0, y_{1}(a)=W_{0} \text {, and } y_{2}(-a)=y_{2}(a)=0 \text {. }
$$

In our simulation, we choose $\lambda(x)=1$, and $W(V)=V$. Define the Hamiltonian

$$
H=u^{2}+\lambda_{1} \mu(x) y_{2}+\lambda_{2} y_{3}+\lambda_{3}\left(-2 u y_{3}-2\right) .
$$

According to the standard result of the optimal control theory, the following costate equations can be obtained:

$$
\left\{\begin{array}{l}
\lambda_{1}^{\prime}(x)=-\frac{\partial H}{\partial y_{1}}=0 \\
\lambda_{2}^{\prime}(x)=-\frac{\partial H}{\partial y_{2}}=-\lambda_{1}(x) \mu(x) \\
\lambda_{3}^{\prime}(x)=-\frac{\partial H}{\partial y_{3}}=2 u(x) \lambda_{3}(x)-\lambda_{2}(x)
\end{array}\right.
$$

with boundary conditions $\lambda_{3}(-a)=\lambda_{3}(a)=0$. The optimal control $u$ is determined by

$$
\begin{aligned}
& \frac{\partial H}{\partial u}=2 u(x)-2 \lambda_{3}(x) y_{3}(x)=0 \\
& \Rightarrow u(x)=\lambda_{3}(x) y_{3}(x) .
\end{aligned}
$$

Generally speaking, there is no analytical solution to the above problem. To solve it numerically, the major difficulty lies in that the boundary conditions are not given at the same point. Here, the classical shooting method [5] is employed to tackle this two-point boundary condition problem. The optimal solutions corresponding to two different $\mu(x)$ are shown in Fig. 3, where the parameters used in the simulation are summarized in Table I. The simulation shows that the solution $V(x)$ is indeed symmetric. Moreover, this example also demonstrates the effect of the proposed weighting functions. When the weighting function decreases from $\mu_{1}(x)$ to $\mu_{2}(x)$, to maintain the weighted average sojourn time at $W_{0}$, a larger $V_{2}(x)$ is resulted.

\section{CONCLUSION}

In this paper, we generalize the optimal sojourn time control (OSTC) problem by introducing weighting functions for both the control cost function and the performance measure function. It is shown that without control weighting, the solution to the 1-D generalized OSTC problem is symmetric. Numerical simulations are performed to verify the results. The way we deal with the performance weighting function does not depend on the state dimension $n$. Therefore, this paper, together with [2], completely proves that without
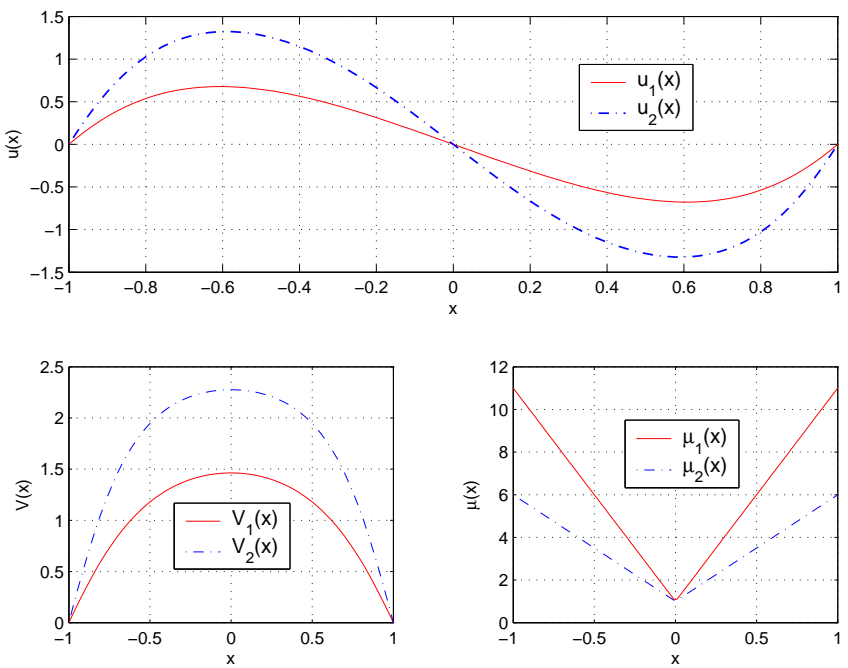

Fig. 3. Simulation Result

control weighting, the solution to an arbitrary dimensional generalized OSTC problem is radially symmetric. Future research will focus on using the the symmetry property to analytically or numerically solve the generalized OSTC problem.

\section{REFERENCES}

[1] J. Hu, and S. Sastry, "Optimal sojourn time control within an interval", Proc. American Control Conference, vol. 4, pp. 3478-3483, Denver, CO, 2003.

[2] J. Hu, "Symmetry of solutions to the optimal sojourn time control problem", Proc. 44 IEEE Int. Conf. Decision and Control, Seville, Spain, 2005

[3] G. Polya and G. Szego, Isoperimetric Inequalities in Mathematical Physics, Princeton Univ. Press, 1951.

[4] R. Raffard, J. Hu, and C. Tomlin, "Adjoint-based optimal control of the expected exit time for stochastic hybrid systems", Hybrid Systems: Computation and Control, 8th Int. Workshop (HSCC 2005), Zurich, Switzerland, pp. 557-572, Springer-Verlag, 2005.

[5] A. E. Bryson and Y. C. Ho, Applied Optimal Control, Taylor and Francis, 1975.

[6] P. Varaiya, "Smart Cars on Smart Roads: Problem of Control", IEEE Transaction on Automatic Control, AC-38(2), 1993.

[7] J. Kuchar and L. C. Yang, "Survey of Conflict Detection and Resolution Modeling Methods", IEEE Transactions on Intelligent Transportation Systems, 1(4):179-189, 2000.

[8] A. Bagchi, Optimal Control of Stochastic Systems, New York, NY: Prentice-Hall, 1993.

[9] B. Oksendal, Stochastic Differential Equations: An Introduction with Applications, Springer-Verlag, 6th edition, 2003

10] C. Bandel, Isoperimetric Inequalities and Applications, Monographs and Studies in Mathematics, vol. 7, Pitman (Advanced Publishing Program), Boston, Mass., 1980

[11] R. O. Saber, W. B. Dunbar, and R. M. Murray, "Cooperative Control of Multi-Vehicle Systems using Cost Graphs and Optimization", Proc. American Contr. Conf., volume 3, pages 2217-2222, Denver, CO, Jun. 2003.

[12] D. Stipanovic, G. Inalhan, R. Teo, and C. J. Tomlin, "Decentralized Overlapping Control of a Formation of Unmanned Aerial Vehicles", Automatica, 40(8):1285-1296, 2004.

[13] W. H. Fleming and R. W. Rishel, Deterministic and Stochastic Optimal Control, New York, NY: Springer-Verlag, 1986. 